\title{
Outcomes of Direct-to-Consumer Hearing Devices for People with Hearing Loss: A Review
}

\author{
Nicole R. $\operatorname{Tran}^{1}$ and Vinaya Manchaiah ${ }^{1,2,3}$ \\ ${ }^{1}$ Department of Speech and Hearing Sciences, Lamar University, Beaumont, TX, USA \\ ${ }^{2}$ Department of Speech and Hearing, School of Allied Health Sciences, Manipal University, Karnataka, India \\ ${ }^{3}$ Audiology India, Mysore, Karnataka, India
}

\author{
Received May 25, 2018 \\ Revised June 6, 2018 \\ Accepted June 7, 2018
}

\section{Address for correspondence Nicole R. Tran, BA Department of Speech and Hearing Sciences, Lamar University, Beaumont, TX 77710, USA \\ Tel +1-951-977-1536 \\ Fax +1-409-880-2265 \\ E-mail ncoffeen@lamar.edu}

\begin{abstract}
As the population of those with mild to moderate hearing loss increases the need remains for amplification solutions. There is a trend to offer alternative amplification options beyond traditional hearing aids. Due to reduced medical risk associated with the most common types of hearing loss (i.e., presbycusis and noise induced), many individuals with such audiological configurations may have success with direct-to-consumer hearing devices (DCHD). The current paper presents a literature review of studies focused on the outcomes of DCHDs for people with hearing loss. Search of electronic databases were used to identify relevant articles for review. Studies on outcomes of DCHDs mainly focused on older adults and reported consistently positive results in clinical and self-reported outcome measures. Improvements in auditory ability, communicative function, social engagement, quality of life, and reduction of self-reported hearing disability were observed. The nature of the direct-to-consumer method makes it challenging to design studies that will accurately represent outcomes for patients due to the extensive dissimilarities in patient journey and device selection options. Accordingly, a majority of the studies conducted on this topic are of low quality of evidence and only provide short-term (i.e., less than one year) outcomes. In addition, results may have been influenced by researcher and/or clinician involvement in choosing the devices and by provision of additional support (i.e., incorporation of a communication partner and communication strategies training). Overall, the literature suggests positive outcomes and self-reported benefit of DCHDs in older adults with hearing loss. However, additional research is needed in this area to verify outcomes.

J Audiol Otol 2018;22(4):178-188
\end{abstract}

KEY WORDS: Hearing aids · Hearing devices · Hearing loss · Over-the-counter hearing aids . Personal sound amplification products · Direct-to-consumer hearing devices.

\section{Introduction}

The World Health Organization (WHO) estimates suggest that 328 million adults have a hearing disability [1]. Of those, roughly 37.5 million adults, $15 \%$ of the United States population have a clinically significant hearing loss [2]. Prevalence of hearing loss increases with age. As such, $25 \%$ of adults between the ages of 65 to 74 have a hearing loss. That rate doubles to $50 \%$ as adults age beyond 75 years or older [3]. According to the 2014 census, "Baby Boomers" will be over the

This is an Open Access article distributed under the terms of the Creative Commons Attribution Non-Commercial License (https://creativecommons.org/licenses/by-nc/4.0/) which permits unrestricted non-commercial use, distribution, and reproduction in any medium, provided the original work is properly cited. age of 65 in the year 2029, at which time they will represent over $20 \%$ of the United States population [4]. The WHO has recognized that untreated hearing loss has an annual global impact of $\$ 250$ billion international dollars [1]. Additionally, it has been shown that the social and emotional effect of a hearing disability can impact an individual's life significantly. Disparities of such impairment include loneliness, social isolation, loss of productivity, and depression [1].

Prevalence of hearing loss is high and will continue to rise with the aging population. Multiple studies suggest that the hearing aid market share has been similar for the last 30 years with uptake rates of around $13-33 \%$ [5-8]. As such, a collective analysis of hearing health care was conducted by the National Institute on Deafness and Other Communication Disor- 
ders/National Institutes of Health (NIDCD/NIH) working group to determine and prioritize future research needs to identify possible solutions to combat this public health dilemma. Specifically, there is a need of intervention options aimed at treatment of age-related hearing loss, clinically known as presbycusis. Several factors including perceived hearing disability, attitude towards hearing aids, cost, and access to hearing healthcare are factors influencing the uptake and use of hearing instruments [9]. Considering the limited insurance (e.g., Medicaid) coverage across the United States for hearing aids [10], it is important to consider new approaches to improve accessibility and affordability of hearing care. Due to reduced medical risk (e.g., ear disorders and acoustic neuroma) associated with the most common types of hearing loss (e.g., presbycusis and noise induced), many individuals with such audiological configurations may have success with direct-to-consumer hearing devices (DCHD). Thus, using DCHD is intended to increase accessibility and affordability of hearing healthcare services by offering devices for these individuals. Higher satisfaction with amplification as reported in the MarkeTrak IX [5] report can be considered increased incentive to find alternative amplification solutions.

The DCHDs are intended to be sold directly to consumers without a consult from a hearing healthcare professional (HCP). The intention of this approach (i.e., direct-to-consumer) is to bypass clinical intervention thereby improving accessibility to amplification and affordability. There are various types of DCHD, including over-the-counter (OTC) hearing devices, personal sound amplification products (PSAPs), and direct-mail-hearing aids (for review see [11]). Previous stipulations by the Food and Drug Administration (FDA) prohibited these devices from being marketed to persons with hearing loss. Instead, these devices were marketed to people with normal hearing with the intention of enhancing hearing and lifestyle; however, many people with hearing loss are using them [12]. As a result of the Over-the-Counter Hearing Aid Act of 2017, these regulations will be revised and there will be a new category of DCHD for OTC hearing aids [13]. These OTC hearing aids will be intended to serve those with mild to moderate hearing loss such as, presbycusis.

As the direct-to-consumer healthcare delivery method is a consumer-focused pathway, there are currently no established guidelines for this approach to hearing healthcare. The standing issue with this delivery model is that when the hearing loss is not noise-related, or age-related, but is instead the result of an underlying medical condition, delivery of an amplification product is not supervised by a trained clinician. The risk therein lies with not receiving treatment for the medical condition that is causing the hearing loss. Additionally, many of the direct-to-consumer devices are programed for a generic sloping hearing loss and are not custom-fit. Therefore, an individual is likely to receive amplification that does not meet their specific hearing thresholds [14].

Studies measuring the electroacoustic characteristics [i.e., 2 cc coupler and simulated gain measurements in Knowles Electronics Manikin for Acoustic Research (GRAS Sound and Vibration, Twinsburg, OH, USA)] of DCHD suggest that most of the devices in the market do not meet the electroacoustic criteria set for hearing aids and do not have the high frequency gain necessary to benefit people with high frequency hearing loss such as, presbycusis [15-18]. However, a recent study reported contrary findings suggesting that despite the heterogeneity among characteristics of devices, certain DCHDs are able to provide appropriate amplification for people with mild to moderate hearing loss [19]. Moreover, in our recent literature review we found evidence to support the hypothesis that DCHDs could potentially benefit people with hearing loss [11]. However, the previous literature review involved loose criteria (i.e., included non-peer reviewed studies, did not perform a quality analysis of the literature) and was not specifically focused on outcomes of DCHD. Therefore, the current paper presents a literature review of studies focusing on the outcomes of DCHD for people with hearing loss. The intention of this review is to expand on the collective studies specifically investigating outcomes of DCHD beyond what was covered in our previous review.

\section{Methods}

A literature search was conducted during April 2017 in the databases CINHAL Complete, MEDLINE, and PsychInfo. Literature search was conducted again during November 2017 to ensure literature review is up-to-date.

\section{Search strategy}

A combination of main and auxillary search words were used to obtain the most applicable search results. The key search words include: over-the-counter hearing device; personal sound amplification product; direct-to-consumer hearing device; direct-to-consumer hearing aid; affordable hearing aid; cheap hearing aid; and cheap hearing device. Auxiliary search words were added to narrow the results. These secondary search words include: hearing; hearing rehabilitation; hearing intervention; and outcomes.

\section{Inclusion criteria}

Due to the novelty of this topic, all articles published in peerreviewed journals were included as long as they met the in- 
clusion criteria, which included: 1) Population - middle aged and older adults, age 50 years or older; 2) Focus - measurements of outcomes (i.e., pre and post device usage) with DCHD; 3) Study type - any study design published in peer-reviewed academic journal; and 4) Language-English. Papers were excluded if they did not meet the inclusion criteria.

A total of 3,220 articles were identified through the electronic database search and 12 additional articles were found through manual search. After the abstract screening, 18 were considered relevant to the topic and full paper was extracted and reviewed. However, only 6 studies were found to meet the inclusion criteria as few studies focusing on electroacoustic characteristics or the consumer surveys were excluded. Fig. 1 represents the flow diagram of the search strategy process.

\section{Quality assessment of studies included}

A quality analysis of the studies was performed using various pre-agreed generic and subject specific criteria. The generic criteria included: 1) having random allocation of subjects; 2) blinding in intervention administration and data collection; 3) having a control group; 4) sample size estimation (or power calculation); 5) having pre-determined inclusion/exclusion criteria; 6) having baseline measures; 7) analysis of bias; 8) effect size reporting; 9) discussion about drop-outs. In addition, domain specific criteria 10) verification of hearing device fit, was also included in the quality analysis of studies.

The studies were rated for quality based on the guidelines from the GRADE Working Group [20,21]. Each of these 10 categories listed above were evaluated for a possibility of two points for meeting each criteria fully resulting in a grand total of 20 possible points. The scoring is as follows:

- $0-5$, Very Low: The results from this study are questionable and uncertain.

- 6-10, Low: Additional research of higher quality may find alternative outcomes with improved study design.

-11-15, Moderate: Additional research may solidify results or change the outcomes observed.

-16-20, High: Additional research is not likely to observe alternative outcomes due to the strength of the existing study.

The results of the quality analysis will be presented in the Results section of this paper.

\section{Results}

\section{Study design, population, and length of trial}

As stated previously, all studies published in peer-reviewed journals were incorporated in this review due to the limited

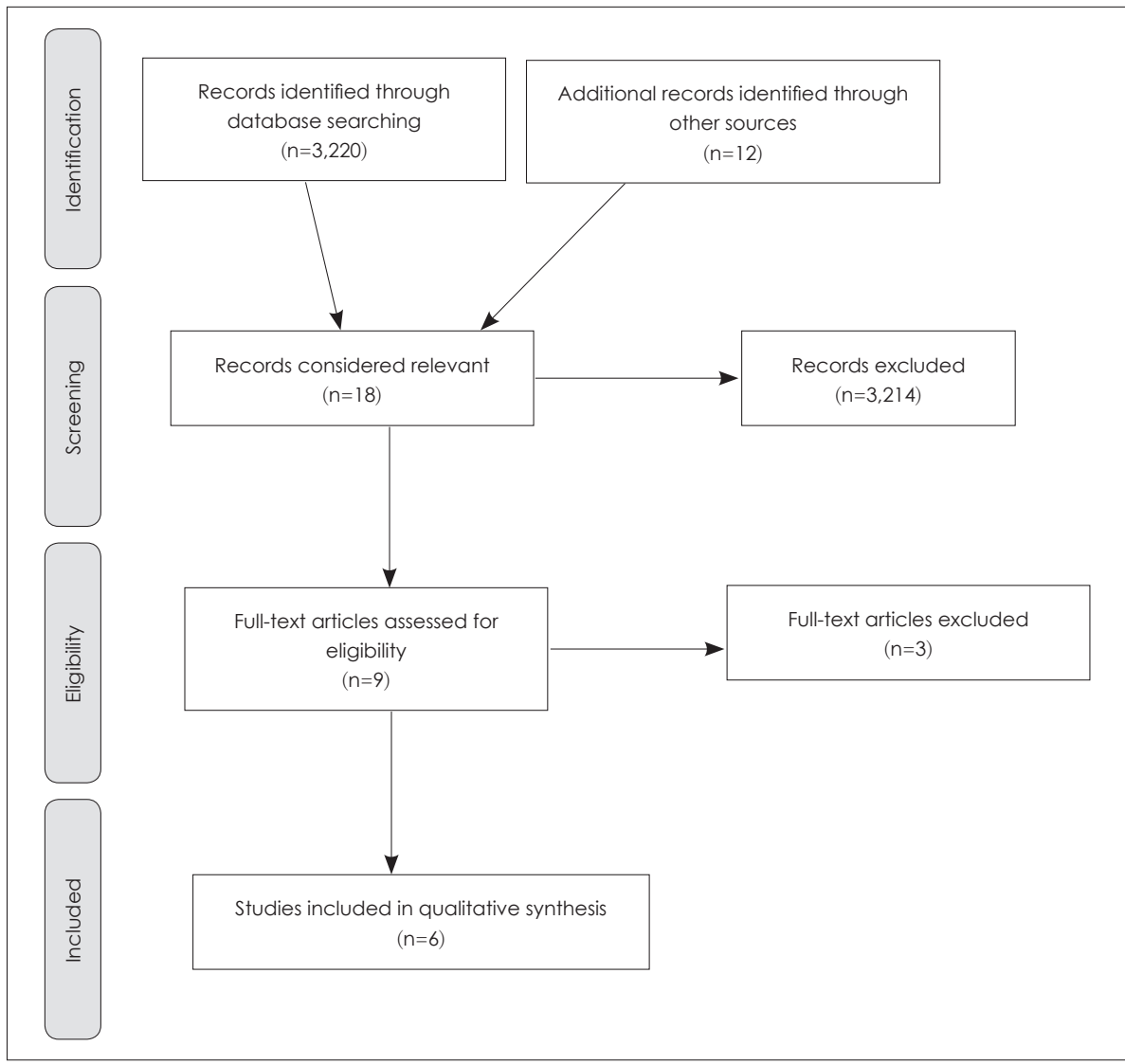

Fig. 1. Flow diagram of the study identification, eligibility search, and inclusion process. 
data, advent of these types of devices, and delivery method (Table 1). It should be noted that there is large variability in study design within the included studies. There are two pilot studies [22,23], two single-group open trials [24,25], one study that utilized randomization and a control group [26] and one study that only measured clinical outcomes [27].

The mean age of participants in the included studies range from 69.1 years old to 78.3 years old. As such, participants presented with the age-related hearing loss configuration known as, presbycusis. The hearing loss is represented by a sloping normal/mild to moderate/severe sensorineural hearing loss in the high frequencies. All studies evaluated participants with presbycusis, but one of the five studies used participants with a comorbidity of a mild cognitive impairment of dementia.

As outlined in Table 1, selected studies varied in their service delivery method. Humes, et al. [26] evaluated the difference between service delivery method of the audiology best practice $(\mathrm{AB})$ approach or the OTC consumer driven (CD) approach. Sacco, et al. [25] and McPherson \& Wong [24] utilized a direct-to-consumer approach with pre-fit devices and limited interaction with a clinician. Mamo, et al. [22] and Nieman, et al. [23] used the HEARS: Hearing Equality through Accessible Research \& Solutions intervention developed in the Nieman, et al. [23] study. The HEARS service delivery method is a single-session intervention with a communication partner (CP) or caregiver which incorporates device selection, device orientation, provision of written materials, and a brief aural rehabilitation (AR) communication strategies training session with CPs. Reed, et al. [27], measured the difference in clinical speech understanding in seven conditions, five of which were with a different PSAP.

There was further variation among the studies regarding length of trial period and daily usage by participants (Table 2). All of the outcomes observed in these studies were short-term (i.e., less than one year). One study only measured outcomes in the clinic, two of the studies were one month long, one was six-weeks long with an optional four-week addition, one was three months, and one was four months. Also, two studies recorded follow-up outcomes for one month-post and 6-monthspost following the completion of intervention. The other four studies had no follow-up measures. Moreover, a majority of the participants used the devices for about an hour a day. However, in the study by Humes, et al. [26], the participants used the device for over 6 hours a day. Briefness in length of trial and low daily usage hinder the generalizability of the proposed positive outcomes.

Table 1. Summary of studies

\begin{tabular}{|c|c|c|c|}
\hline Source/country & Study design & Population & Summary of outcomes \\
\hline $\begin{array}{l}\text { Humes, et al. [26] } \\
\text { USA }\end{array}$ & $\begin{array}{l}\text { Prospective, quantitative, } \\
\text { randomized, double- } \\
\text { blind, placebo- } \\
\text { controlled trial with } \\
\text { three categories }\end{array}$ & $\begin{array}{l}\mathrm{n}=154 ; \text { mean age } 69.1 \text { yrs } \\
\text { (across all } 3 \text { groups) }\end{array}$ & $\begin{array}{l}\text { Outcomes show benefit of either type of } \\
\text { amplification regardless of delivery method. } \\
\text { Participants with no gain hearing aids did not } \\
\text { have same benefits. }\end{array}$ \\
\hline $\begin{array}{l}\text { Sacco, et al. [25] } \\
\text { France }\end{array}$ & $\begin{array}{l}\text { Prospective, quantitative, } \\
\text { single focus (group) }\end{array}$ & $\mathrm{n}=31$; mean age 78.3 yrs & $\begin{array}{l}\text { Improvements of quality of life, decreased } \\
\text { hearing difficulties, reduced negative emotion } \\
\text { for background noise, and enhanced } \\
\text { conversations in noise. }\end{array}$ \\
\hline $\begin{array}{l}\text { Nieman, et al. [23] } \\
\text { USA }\end{array}$ & $\begin{array}{l}\text { Pilot study, prospective, } \\
\text { quantitative, } \\
\text { randomized, control } \\
\text { group }\end{array}$ & $\begin{array}{l}\mathrm{n}=15 \text { dyads; participants } \\
\text { mean age } 70.1 \mathrm{yrs} \text {; } \\
\text { communication partner } \\
\text { at least } 18 \text { yrs old }\end{array}$ & $\begin{array}{l}\text { Disease specific improvements (hearing } \\
\text { handicap), but generic quality of life was } \\
\text { unchanged. }\end{array}$ \\
\hline $\begin{array}{l}\text { Mamo, et al. [22] } \\
\text { USA }\end{array}$ & $\begin{array}{l}\text { Pilot study, prospective, } \\
\text { quantitative, qualitative }\end{array}$ & $\begin{array}{l}\text { n=20 dyads; participants } \\
\text { mean age } 76.9 \text { yrs } \\
\text { with mild cognitive } \\
\text { impairment-dementia; } \\
\text { caregivers mean age } \\
64.3 \mathrm{yrs}\end{array}$ & $\begin{array}{l}\text { No significant objective measures of } \\
\text { improvements, but caregivers provided } \\
\text { qualitative response of improved } \\
\text { communication. }\end{array}$ \\
\hline $\begin{array}{l}\text { McPherson \& Wong [24] } \\
\text { Hong Kong }\end{array}$ & $\begin{array}{l}\text { Prospective, quantitative, } \\
\text { qualitative }\end{array}$ & $\mathrm{n}=19$; mean age 73 yrs & $\begin{array}{l}\text { Slight improvement of quality of life, but open } \\
\text { interviews provided mixed positive/negative } \\
\text { feedback. }\end{array}$ \\
\hline $\begin{array}{l}\text { Reed, et al. [27] } \\
\text { USA }\end{array}$ & Prospective, quantitative & $\mathrm{n}=42 ;$ mean age 71.6 & $\begin{array}{l}\text { Some personal sound amplification products } \\
\text { showed improved clinical measures of speech } \\
\text { understanding when compared to unaided. }\end{array}$ \\
\hline
\end{tabular}


Table 2. Daily use of direct-to-consumer hearing devices and length of study

\begin{tabular}{lcl}
\hline \multicolumn{1}{c}{ Source } & Average daily use (hr) & \multicolumn{1}{c}{ Study length } \\
\hline Humes, et al. [26] & 6.3 & 6-weeks; with additional 4-weeks \\
Sacco, et al. [25] & 1 & One month \\
Nieman, et al. [23] & $1-4$ & Three months; with 3 \& 6-month follow-up \\
Mamo, et al. [22] & 1 & One month; with a one month follow-up \\
McPherson \& Wong [24] & $1-8$ & Four months; with measures taken throughout \\
Reed, et al. [27] & N/A & Clinical only \\
\hline
\end{tabular}

Table 3. Outcome measures

\begin{tabular}{|c|c|c|c|}
\hline Source & Baseline measures & Outcome measures (i.e., pre- and post) & Post-intervention measures \\
\hline Humes, et al. [26] & $\begin{array}{l}\text { - PTA } \\
\text { - Speech Recognition Threshold (SRT) } \\
\text { - Word Recognition Scores in Quiet } \\
\text { (WRS-Q) } \\
\text { - Loudness Discomfort Level (LDL) } \\
\text { - Mini-Mental State Exam-2nd } \\
\text { Edition (MMSE-2 SV) }\end{array}$ & $\begin{array}{l}\text { - Profile of Hearing Aid Benefit (PHAB) } \\
\text { - Hearing Handicap Inventory for } \\
\text { Elderly (HHIE) } \\
\text { - Connected Speech Test (CST) }\end{array}$ & $\begin{array}{l}\text { - Hearing Aid Satisfaction } \\
\text { Survey (HASS) } \\
\text { - Practical Hearing Aid Skills } \\
\text { Test-Revised (PHAST-R) }\end{array}$ \\
\hline Sacco, et al. [25] & $\begin{array}{l}\text { - } \text { PTA } \\
\text { - } \text { Mini-Mental State Examination } \\
\text { (MMSE) } \\
\text { - Instrumental Activities of Daily } \\
\text { Living for Elderly (IADL-E) }\end{array}$ & $\begin{array}{l}\text { - } \text { PTA } \\
\text { - Speech Audiometry } \\
\text { - Acceptable Noise Level (ANL) } \\
\text { - Glasgow Hearing Ai Benefit Profile } \\
\text { (GHABP) }\end{array}$ & $\begin{array}{l}\text { - Acceptability of device } \\
\text { measures-Custom survey }\end{array}$ \\
\hline $\begin{array}{l}\text { Nieman, et al. } \\
\text { [23] }\end{array}$ & $\begin{array}{l}\text { - } \text { PTA } \\
\text { - Rapid Estimate of Adult Literacy in } \\
\text { Medicine (REALM-R) } \\
\text { - Montreal Cognitive Assessment } \\
\text { (MoCA) } \\
\text { - Hearing-related self-efficacy- } \\
\text { The Line from the Ida Institute } \\
\text { - Attitudes Toward Computers Ques- } \\
\text { tionnaire (ATCQ) }\end{array}$ & $\begin{array}{l}\text { - Hearing Handicap Inventory for } \\
\text { Elderly-Screening (HHIE-S) } \\
\text { - Quantified Denver Scale } \\
\text { of Communication Function (QDS) } \\
\text { - Revised UCLA Loneliness Scale } \\
\text { - Patient Health Questionnaire-9 (PHQ-9) } \\
\text { - Short-Form General Health Survey-36 } \\
\text { items (SF-36) }\end{array}$ & $\begin{array}{l}\text { - International Outcome } \\
\text { Inventory-Alternative } \\
\text { Interventions (IOI-Al) } \\
\text { - Self-reported willingness to } \\
\text { pay-Custom survey }\end{array}$ \\
\hline Mamo, et al. [22] & $\begin{array}{l}\text { - PTA } \\
\text { - Mini-Mental State Examination } \\
(\text { MMSE })\end{array}$ & $\begin{array}{l}\text { - Cornell Scale for Depression } \\
\text { in Dementia (CSDD) } \\
\text { - Neuropsychiatric Inventory- } \\
\text { Questionnaire (NPI-Q) } \\
\text { - Zarit Burden Interview (ZBI)-Caregiver } \\
\text { burden } \\
\text { - International Outcome Inventory- } \\
\text { Alternative Intervention-Significant } \\
\text { Other (IOI-AI-SO) }\end{array}$ & $\begin{array}{l}\text { - Qualitative data from } \\
\text { participants and } \\
\text { caregivers }\end{array}$ \\
\hline $\begin{array}{c}\text { McPherson \& } \\
\text { Wong [24] }\end{array}$ & - PTA & $\begin{array}{l}\text { - Objective measures of hearing aid } \\
\text { output (i.e., insertion gain) } \\
\text { - Client-Oriented Scale of Improvement } \\
\text { (COSI) } \\
\text { - International Outcome Inventory- } \\
\text { Hearing Aids (IOI-HA) } \\
\text { - Profile of Hearing Aid Performance-- } \\
\text { Chinese Version (PHAP-C) }\end{array}$ & - Open-ended interviews \\
\hline Reed, et al. [27] & $\begin{array}{l}\text { - Mini-Mental State Examination } \\
\text { (MMSE) } \\
\text { - AZBio }\end{array}$ & - AZBio & - N/A \\
\hline
\end{tabular}

PTA: pure-tone average 
Baseline, outcome, and post-intervention measures

The studies used various types of baseline, outcome and post-intervention measures (Table 3 ). The baseline measures were mainly clinical measures such as pure-tone average (PTA), speech testing, and acceptable noise level (ANL) assessment. The self-reported baseline measures included: mental-state, activities of daily living, hearing-related self-efficacy, health literacy and attitude towards computers. Outcome measures included both clinical measures and self-reported measures. The study by Sacco, et al. [25] used PTA, speech audiometry and ANL assessment as outcome measures. The study by Humes, et al. [26] and McPherson \& Wong [24] used Connected Speech Test (CST) and hearing aid insertion gain (i.e., hearing aid output) as clinical outcome measures, respectively. Reed, et al. [27] used the AZBio as the only clinical measure of outcome. With the exception of Reed, et al. [27], the other five studies utilized self-reported outcome measures for patients. Additionally, the study by Mamo, et al. [22] utilized self-reported outcome measures administered to caregivers (i.e., caregiver burden and communication function). Self-reported outcome measures of patients measured the following constructs: hearing aid outcome, hearing disability, communication function, depression, neuropsychiatric symptom, and quality of life (Table 4). Likewise, most of the studies used additional post-intervention measures. These measures focused on hearing device satisfaction, hearing device-handling skills, acceptability of the device, willingness to pay, and qualitative perspectives of users and their caregivers.

\section{Types of devices and amplification settings}

As shown in Table 5, a variety of devices were used within these studies. Device types included traditional hearing aids (approved by the FDA), OTC hearing devices, PSAPs, and assistive listening devices (ALDs). Nieman, et al. [23] and Mamo, et al. [22] used the same devices because the two studies implemented the same delivery method with low-income urban dwelling adults and mild cognitively impaired adults, respectively. The participants in these two studies had the option to choose between the Sound World Solutions CS50 (Park Ridge, IL, USA) monaural PSAP device and the Williams Sound Pocketalker Ultra Duo Pack (Eden Praire, MN, USA) which is an ALD with binaural over-the-ear headphones. These two devices are quite different in appearance and features. The CS-50 looks like a mobile phone Bluetooth device by housing all of the components within the ear piece. The Pocketalker ALD makes use of a microphone and wired headphones. Alternatively, McPherson \& Wong [24] used a ReSound Avance HE4 (Bloomington, MN, USA) OTC behind-the-ear device that was discontinued in the United States in the early 2000's. This device can be custom fit to an individual's hearing loss, but for the purposes of the study, it was programed for a predetermined configuration with default sound output to represent the typical OTC delivery method. Sacco, et al. [25] used a newly designed OTC product by Tinteo-Personal Sound ${ }^{\odot}$ Society in Meyreuil, France, called the TEO First ${ }^{\circledR}$. This product uses a digital signal-processing (DSP) chip for advanced signal processing capabilities such as dynamic compression. Considering the American National

Table 4. Constructs of outcome metrics

\begin{tabular}{|c|c|c|c|c|c|c|}
\hline \multirow[b]{2}{*}{ Outcome metrics } & \multicolumn{5}{|c|}{ Studies } & \multirow[b]{2}{*}{$\begin{array}{c}\text { Reed, et al. } \\
\text { [27] }\end{array}$} \\
\hline & $\begin{array}{c}\text { Humes, et al. } \\
{[26]}\end{array}$ & $\begin{array}{c}\text { Sacco, et al. } \\
{[25]}\end{array}$ & $\begin{array}{c}\text { Nieman, et al. } \\
\text { [23] }\end{array}$ & $\begin{array}{c}\text { Mamo, et al. } \\
\text { [22] }\end{array}$ & $\begin{array}{c}\text { McPherson \& } \\
\text { Wong [24] }\end{array}$ & \\
\hline \multicolumn{7}{|l|}{ Patient specific } \\
\hline \multicolumn{7}{|l|}{ Clinical } \\
\hline Pure-tone audiometry & & $\checkmark$ & & & & \\
\hline Speech audiometry & $\checkmark$ & $\checkmark$ & & & & $\checkmark$ \\
\hline Acceptable noise level & & $\checkmark$ & & & & \\
\hline Insertion gain & & & & & $\checkmark$ & \\
\hline \multicolumn{7}{|l|}{ Self-reported } \\
\hline Hearing aid outcome & $\checkmark$ & $\checkmark$ & & & $\checkmark$ & \\
\hline Hearing disability & $\checkmark$ & & $\checkmark$ & & & \\
\hline Communication function & & & $\checkmark$ & & & \\
\hline Depression & & & $\checkmark$ & $\checkmark$ & & \\
\hline Neuropsychiatric symptoms & & & & $\checkmark$ & & \\
\hline Quality of Life & & & $\checkmark$ & & & \\
\hline \multicolumn{7}{|l|}{ Caregiver specific (self-reported) } \\
\hline Caregiver burden & & & & $\checkmark$ & & \\
\hline Communication function & & & & $\checkmark$ & & \\
\hline
\end{tabular}


Table 5. Devices, type, cost, and features

\begin{tabular}{|c|c|c|c|c|}
\hline Source & Device used & Cost & Device type & Device features \\
\hline $\begin{array}{l}\text { Humes, et al. } \\
\text { [26] }\end{array}$ & $\begin{array}{l}\text { ReSound Alera } 9 \\
\text { Mini-BTE } \\
\text { (Bloomington, MN, } \\
\text { USA) }\end{array}$ & $\$ 100$ each & $\begin{array}{l}\text { FDA approved } \\
\text { hearing aid }\end{array}$ & $\begin{array}{l}\text { Advanced signal processing: multi-channel } \\
\text { compression; feedback cancellation; noise } \\
\text { reduction; directional microphones; four } \\
\text { programs }\end{array}$ \\
\hline Sacco, et al. [25] & $\begin{array}{l}\text { TEO First® (Meyreuil, } \\
\text { France) }\end{array}$ & $\$ 250$ each & $\begin{array}{l}\text { OTC developed in } \\
\text { France }\end{array}$ & $\begin{array}{l}\text { Amplification range } 0-22 \mathrm{~dB} \text {; digital signal } \\
\text { processing; multiband dynamic compression; } \\
\text { two programs (calm \& noisy); volume } \\
\text { control; rechargeable batteries }\end{array}$ \\
\hline $\begin{array}{l}\text { Nieman, et al. } \\
\text { [23] }\end{array}$ & $\begin{array}{l}\text { Sound World Solutions } \\
\text { CS-50 (Park Ridge, IL, } \\
\text { USA); Williams Sound } \\
\text { Pocketalker Ultra Duo } \\
\text { Pack (Eden Praire, } \\
\text { MN, USA) }\end{array}$ & $\begin{array}{l}\$ 350(\text { CS-50); } \\
\$ 120 \\
\quad \text { (Pocketalker) }\end{array}$ & $\begin{array}{l}\text { CS-50: PSAP; } \\
\text { Pocketalker: } \\
\text { ALD }\end{array}$ & $\begin{array}{l}\text { CS-50: monaural device, Bluetooth, paired \& } \\
\text { programmed with smartphone, rechargeable } \\
\text { batteries; } \\
\text { Pocketalker: remote microphone, } \\
\text { headphones, volume control, AAA batteries, } \\
\text { non-programmable }\end{array}$ \\
\hline Mamo, et al. [22] & $\begin{array}{l}\text { Sound World Solutions } \\
\text { CS-50; Williams Sound } \\
\text { Pocketalker Ultra Duo } \\
\text { Pack }\end{array}$ & $\$ 100-\$ 300$ & $\begin{array}{l}\text { CS-50: PSAP; } \\
\text { Pocketalker: } \\
\text { ALD }\end{array}$ & $\begin{array}{l}\text { CS-50: monaural device, Bluetooth, paired \& } \\
\text { programmed with smartphone, rechargeable } \\
\text { batteries; } \\
\text { Pocketalker: remote microphone, } \\
\text { headphones, volume control, AAA batteries, } \\
\text { non-programmable }\end{array}$ \\
\hline $\begin{array}{c}\text { McPherson \& } \\
\text { Wong [24] }\end{array}$ & $\begin{array}{l}\text { ReSound Avance HE4 } \\
\text { (Bloomington, MN, } \\
\text { USA) }\end{array}$ & $\$ 125$ each & $\begin{array}{l}\text { Hearing aid sold } \\
\text { as OTC in China/ } \\
\text { Hong Kong }\end{array}$ & $\begin{array}{l}\text { Max gain of } 31 \mathrm{~dB} \text { SPL; semi-open-canal fit; } \\
\text { size } 10 \text { batteries; sound compression and } \\
\text { volume trimmer }\end{array}$ \\
\hline Reed, et al. [27] & $\begin{array}{l}\text { Sound World Solutions } \\
\text { CS-50, Soundhawk } \\
\text { (Cupertino, CA, USA), } \\
\text { Etymotic BEAN (Elk } \\
\text { Grove Village, IL, } \\
\text { USA), Tweak Focus } \\
\text { (Johnson City, TN, } \\
\text { USA), and MSA 30X } \\
\text { Sound Amplifier (Van } \\
\text { Nuys, CA, USA) }\end{array}$ & $\$ 30-\$ 350$ & PSAPs sold in USA & $\begin{array}{l}\text { CS-50: monaural device, Bluetooth, paired \& } \\
\text { programmed with smartphone, rechargeable } \\
\text { batteries; } \\
\text { Soundhawk: Bluetooth, programmable with } \\
\text { smartphone, rechargeable, no longer sold in } \\
\text { USA; } \\
\text { BEAN: in-ear amplifier, tele-coil option, non- } \\
\text { programmable; } \\
\text { Tweak Focus: behind-the-ear device, tele-coil } \\
\text { option, three volumes, non-programmable; } \\
\text { MSA 30X: behind-the-ear device, available } \\
\text { at retail stores, rechargeable, non- } \\
\text { programmable }\end{array}$ \\
\hline
\end{tabular}

FDA: Food and Drug Administration, OTC: over-the-counter, ALD: assistive listening device, PSAPs: personal sound amplification products

Standards Institute (ANSI) standards enforced by the FDA, the highest performing device is the ReSound Alera 9 MiniBTE (Bloomington, MN, USA)device used in the Humes, et al. [26] study. This device has full DSP advanced signal processing capabilities such as multi-channel compression, feedback cancelation, noise reduction, and directional microphones. Alternatively, Reed, et al. [27] used the most comprehensive collection of PSAPs by incorporating five devices: Sound World Solutions CS-50, Soundhawk (Cupertino, CA, USA), Etymotic BEAN (Elk Grove Village, IL, USA), Tweak Focus (Johnson City, TN, USA), and MSA 30X Sound Am- plifier (Van Nuys, CA, USA).

In addition to product variations, each study utilized the features of the devices differently (Table 6). The Humes, et al. [26], having the highest quality device, utilized four different programs for the participants. The group that received the AB intervention offered a custom fit device to National Acoustic Laboratories Non-Linear Prescription Formula, Version 2 (NAL-NL2) prescriptive fitting targets. The group that received the $\mathrm{CD}$ approach was able to select from one of three devices with pre-programed amplification settings. The selections were programed for generic configurations that repre- 
Table 6. Monaural vs. binaural fitting, prescriptive fitting formula, and verification of the device

\begin{tabular}{llll}
\hline \multicolumn{1}{c}{ Source } & \multicolumn{1}{c}{ Verification of device fit } & \multicolumn{1}{c}{ Prescriptive fitting type } & Fitting \\
\hline Humes, et al. [26] & Yes & AB: custom fit NAL-NL2; & Binaural \\
Sacco, et al. [25] & No & Pre-fit to common HL & Binaural \\
Nieman, et al. [23] & No & PSAP: fit according to cell phone program response; & PSAP: monaural; \\
& & ALD: volume control only & ALD: binaural \\
Mamo, et al. [22] & No & PSAP: fit according to cell phone program response; PSAP: monaural; \\
& & ALD: volume control only & ALD: binaural \\
McPherson \& Wong [24] & Yes & NAL-R & Monaural \\
Reed, et al. [27] & Yes & Unspecified & Monaural \\
\hline AB: audit
\end{tabular}

AB: audiology best practice, CD: consumer driven, NAL-NL2: National Acoustic Laboratories Non-Linear Prescription Formula, Version 2, HL: hearing loss, PSAP: personal sound amplification product, ALD: assistive listening device, NAL-R: National Acoustics Laboratory-Revised

sented either a mild, moderate, or severe presbycusis sloping hearing loss. The placebo group in the study received devices set to $0 \mathrm{~dB}$ gain. Alternatively, Sacco, et al. [25] used the TEO First $^{\circledR}$ OTC device with a pre-programed sloping presbycusis hearing loss. These participants were able to make use of two programs. The first program, intended for use in quiet environments, was programed to the average hearing loss thresholds for a 65-year-old man. The second program, intended for use in more noisy environments, was programed to meet the average hearing thresholds for a 75 -year-old man. Conversely, Nieman, et al. [23] and Mamo, et al. [22] gave participants the option to select from the CS-50 (monaural PSAP) or the Pocketalker (binaural ALD). The CS-50 can be programed to the patient's auditory preferences with the use of a mobile app. The Pocketalker has a volume control, but does not have any acoustic programing features-it simply amplifies all incoming sounds. McPherson \& Wong [24] used a device that could be custom fit, but instead programed the devices to the default sound output for a common presbycusis hearing loss configuration to match National Acoustics Laboratory-Revised (NAL-R) targets. Studies by Humes, et al. [26] and McPherson \& Wong [24] did perform hearing aid verification using real-ear measures. Reed, et al. [27] reports using best-practice verification to match devices to better-hearing ear. It is unclear what type of verification was performed considering the lack of programmability and customization available with the PSAP devices.

The studies that present electroacoustic data of DCHD suggest that nearly $90 \%$ of the devices do not meet the strict criteria set for hearing aids (for review see [11]). Also, the devices used in many of these studies do not adequately represent the abundant products available as direct-to-consumer options. Moreover, in all the studies the researcher (i.e., trained hearing HCP) chose the device. This may have introduced selection bias by not representing how a typical non-
HCP would purchase a DCHD in the open market. Additionally, the concentration of these outcome measures is intended for direct-to-consumer devices, specifically PSAPs and OTCs, not ALDs. However, in Nieman, et al. [23] and Mamo, et al. [22] a PSAP and ALD are used. Since neither of these studies revealed how many participants selected the PSAP versus the ALD, outcomes may be related to ALD use, which is outside the scope of our aim. Conversely, Humes, et al. [26] used an FDA approved hearing aid device. This device offers advanced signal processing capabilities beyond that of common OTC devices in the United States. It is plausible that a high-end device may positively influence outcomes due to better electroacoustic characteristics $[11,18]$. Sacco, et al. [25] and Reed, et al. [27] used OTCs and PSAPs that more accurately represent the market options.

\section{Service delivery model and additional support}

The divergence of delivery method and the provision of additional support impact the uniformity of study design and outcomes (Table 7). Key influences may include the involvement of a trained clinician delivering the device orientation, communication strategies training, and incorporation of a CP. All three of these aid in improved outcomes independently but are not components of a traditional direct-to-consumer approach. One of the pillars of the OTC delivery method is to improve accessibility; however, the involvement of a clinician providing device support and AR are alternatively hallmarks of audiology best practice evidence-based approach. Moreover, support from a CP to be equally trained on device manipulation and communication strategies could greatly improve the patient's acceptance of the delivery method, device, and improve outcomes. As the provision of additional support does not usually accompany the DCHD delivery method, the reported outcomes may not reflect the accuracy of the patient journey in open market. 
Table 7. Service delivery method and additional support

\begin{tabular}{ccc}
\hline Source & \multicolumn{1}{c}{ Service delivery method } & Provision of additional support \\
\hline Humes, et al. [26] & AB: Audiology Best Practice method & AB\&P: device orientation, communication \\
& CD: OTC self-select, pre-programed acoustic settings & strategies training; CD: OTC method, no \\
& P: Audiology Best Practice, but hearing aid programmed to & additional clinician support \\
& O dB gain & \\
Sacco, et al. [25] & Pre-selected device with pre-fitted hearing loss configurations; Device orientation of OTC, no additional \\
& most similar to OTC delivery method. & clinician support \\
Nieman, et al. & HEARS Program. Community based, single-session delivery & Device orientation, Communication strate- \\
[23] & method with clinician. Selection between PSAP or ALD. & gies AR training, Incorporation of CP \\
Mamo, et al. [22] & HEARS Program. Community based, single-session delivery & Device orientation, communication strate- \\
& method with clinician. Selection between PSAP or ALD. & gies AR training, incorporation of CP \\
McPherson \& & Single device option, pre-fit to common hearing loss & OTC: no additional clinician support \\
Wong [24] & configuration to simulate OTC delivery model. & \\
Reed, et al. [27] & Devices adjusted to fit hearing in the better ear. & N/A \\
\hline AB:
\end{tabular}

AB: audiology best practice, CD: consumer driven, OTC: over-the-counter, HEARS: Hearing Equality through Accessible Research \& Solutions, ALD: assistive listening device, PSAPs: personal sound amplification products, CP: communication partner, AR: aural rehabilitation

Table 8. Quality assessment of studies included

\begin{tabular}{lccccccccccc}
\hline \multicolumn{1}{c}{ Source } & RCT & $\begin{array}{c}\text { Double- } \\
\text { bind RCT }\end{array}$ & $\begin{array}{c}\text { Control } \\
\text { group }\end{array}$ & $\begin{array}{c}\text { Incl/ } \\
\text { excl }\end{array}$ & Baseline & $\begin{array}{c}\text { Verify } \\
\text { HA Fit }\end{array}$ & $\begin{array}{c}\text { Analysis } \\
\text { bias }\end{array}$ & $\begin{array}{c}\text { Effect } \\
\text { size }\end{array}$ & $\begin{array}{c}\text { Power } \\
\text { analysis }\end{array}$ & $\begin{array}{c}\text { Drop- } \\
\text { out }\end{array}$ & $\begin{array}{c}\text { Score/level } \\
\text { of evidence }\end{array}$ \\
\hline Humes, et al. [26] & 2 & 2 & 2 & 2 & 2 & 2 & 2 & 2 & 2 & 2 & $20:$ High \\
Sacco, et al. [25] & 0 & 0 & 0 & 2 & 2 & $1^{*}$ & $1^{\dagger}$ & 0 & 0 & 2 & 8: Low \\
Nieman, et al. [23] & 2 & 0 & 2 & 2 & 2 & 0 & 0 & 2 & 0 & 0 & 10: Low \\
Mamo, et al. [22] & 0 & 0 & 0 & 2 & 2 & 0 & 0 & 0 & 0 & 2 & 6 6: Low \\
McPherson \& Wong [24] & 0 & 0 & 0 & 0 & 2 & 2 & 0 & 0 & 0 & 2 & 6: Low \\
Reed, et al. [27] & 0 & 0 & 0 & $1^{*}$ & 2 & $1^{*}$ & 0 & 0 & 0 & 0 & 3: Low \\
\hline
\end{tabular}

*unspecified type of verification, ${ }^{\dagger}$ independent study, but funded by the manufacturer, ${ }^{\dagger}$ configuration of hearing loss is unclear. RCT: randomized control trial, HA: hearing aid

\section{Quality analysis}

The quality analysis performed (Table 8), shows that all but one of the studies conducted in this area are considered to be of low quality. The study by Humes, et al. [26] has the highest level of evidence with a score of 20 indicating that additional research is unlikely to reveal alternative outcomes. This study was the first double-blind randomized control trial (RCT) with a placebo group study completed in this area of research. Sacco, et al. [25], Nieman, et al. [23], Mamo, et al. [22], Reed, et al. [27], and McPherson \& Wong [24] scored within the low ranking quality of evidence. Additional research in this area is highly recommended to verify outcomes. Nevertheless, the Nieman, et al. [23] and Mamo, et al. [22] studies were conducted as pilot studies, which alleviates the expectation for a rigorous, high-quality design. Therefore, these two were appropriately designed for their purpose as pilot studies.

\section{Discussion}

Summary of main findings, study limitations, and future directions

There is consensus of positive outcomes observed in each study. All studies that measured self-reported outcomes observed: reduction of self-perceived disability, improved communication function, improved hearing aid outcome, and increased social engagement (Table 9). The agreement of positive outcomes observed is motivational for further research to be conducted. Nevertheless, recall from the quality analysis that five of the six studies presented have low quality of evidence. More robust study designs should be implemented. Furthermore, key weaknesses that impede the acceptance and generalizations of results include: 1) variations in device options; 2) dissimilarities in delivery method; 3) consistently brief trial periods; and 4) provision of additional support by clinician and/or CP. In all studies, the researchers, who have specialist knowledge of amplification, selected the devices. While this may be considered appropriate to control for variances, a majority of the devices selected are not representative of devices on the market (with the exception of Reed, et al. [27]). The true DCHD service-delivery pathway is the direct purchase of a device, from a plethora of options on the free market, by an individual with limited and/or no knowledge of hearing devices. Also, the currently published reports provide results of short-term outcomes (i.e., less than one year). 
Considering that the DCHD are of less cost and potentially of less electroacoustic quality [11], it can be speculated that these devices may not last for very long or may not have the same characteristics in the longer term due to usage. Consequently, we do not know the long-term (i.e., 12 months or longer) outcome of these devices. While the provision of additional sup- port and possible inclusion of a $\mathrm{CP}$ is considered best practice, it does not reflect the pathway of the DCHD model. Hence, these studies may not provide realistic outcomes of the direct-to-consumer approach by deviating from the service delivery method. Also, in the case of Reed, et al. [27], restriction of outcomes to the clinical setting prevented the pa-

Table 9. Summary of results and limitations

\begin{tabular}{|c|c|c|}
\hline Source & Summary of results & Limitations \\
\hline Humes, et al. [26] & $\begin{array}{l}\text { - AB \& CD groups had better outcomes than P group } \\
\text { indicating benefit of amplification in general. } \\
\text { - Daily usage of device was not influenced by delivery } \\
\text { method. } \\
\text { - Price influenced desire to keep device at the end of trail, } \\
\text { but did not influence amount of daily usage. }\end{array}$ & $\begin{array}{l}\text { - Use of high-end device may over represent } \\
\text { positive outcomes due to superior } \\
\text { electroacoustic abilities. } \\
\text { - Results may not be generalized to other } \\
\text { devices. }\end{array}$ \\
\hline Sacco, et al. [25] & $\begin{array}{l}\text { - Improvement in: speech and puretone thresholds, } \\
\text { quality of life measures, conversation with/without noise \& } \\
\text { with TV. } \\
\text { - Decreased hearing difficulties and decreased negative } \\
\text { emotion relating to presence of background noise. } \\
\text { - Acceptability of device was low to moderate. }\end{array}$ & $\begin{array}{l}\text { - Limited device selection } \\
\text { - Absence of a control group } \\
\text { - Low acceptability of the device } \\
\text { - Short-term measure of outcomes with a one- } \\
\text { month trial and low daily usage of } 60 \text { minutes }\end{array}$ \\
\hline $\begin{array}{l}\text { Nieman, et al. } \\
\text { [23] }\end{array}$ & $\begin{array}{l}\text { - Improvement of disease-specific outcomes such as } \\
\text { hearing handicap and communication function. } \\
\text { - Reduction of depressive symptoms. } \\
\text { - Generic quality of life outcomes were primarily } \\
\text { inconclusive or unchanged (i.e., loneliness). } \\
\text { - Willingness to pay for HEARS program with device: } \$ 87.50 \text {. }\end{array}$ & $\begin{array}{l}\text { - Pilot study-small sample size \& short-term } \\
\text { outcome measures: results cannot be } \\
\text { generalized. } \\
\text { - Limited device selection } \\
\text { - Involvement of clinician/CP and AR may have } \\
\text { inflated positive outcomes. } \\
\text { - Unspecified amount of participants used PSAP } \\
\text { vs. ALD. }\end{array}$ \\
\hline Mamo, et al. [22] & $\begin{array}{l}\text { - No significant change was measured for CSDD, NPI-Q or } \\
\text { ZBI. } \\
\text { - Qualitative responses from caregivers reported increased } \\
\text { communicative benefit of patient. } \\
\text { - Patients with lowest depressive scores showed the } \\
\text { greatest improvement. } \\
\text { - Research claims benefit of amplification as a } \\
\text { nonpharmacologic option for treating depression and } \\
\text { neuropsychiatric symptoms in dementia patients. }\end{array}$ & $\begin{array}{l}\text { - Pilot study-small sample size \& short-term } \\
\text { outcome measures: results cannot be } \\
\text { generalized. } \\
\text { - Limited device selection } \\
\text { - Involvement of clinician/CP and AR may have } \\
\text { inflated positive outcomes. } \\
\text { - Unspecified amount of participants used PSAP } \\
\text { vs. ALD. }\end{array}$ \\
\hline $\begin{array}{c}\text { McPherson \& } \\
\text { Wong [24] }\end{array}$ & $\begin{array}{l}\text { - Slight improvement in QOL. } \\
\text { - Outcomes improved as length of trial increased } \\
\text { indicating more time with the hearing aid usage } \\
\text { improved scores. } \\
\text { - Open interviews were mixed with negative focus on } \\
\text { presence of noise with device (i.e., background or } \\
\text { feedback). }\end{array}$ & $\begin{array}{l}\text { - No control group } \\
\text { - Single/limited device selection } \\
\text { - Short-term outcome measures }\end{array}$ \\
\hline Reed, et al. [27] & $\begin{array}{l}\text { - Speech understanding increased with four out of five } \\
\text { PSAPs with an improvement from } 76.5 \% \text { unaided, to } 88.4 \% \\
\text { aided. }\end{array}$ & $\begin{array}{l}\text { - Unclear if patients had a bilateral, unilateral, } \\
\text { or asymmetrical hearing loss. } \\
\text { - Clinical measures only } \\
\text { - Small/convenience sample } \\
\text { - Does not follow DCHD model with self-fitting. }\end{array}$ \\
\hline
\end{tabular}

AB: audiology best practice, CD: consumer driven, HEARS: Hearing Equality through Accessible Research \& Solutions, CSDD: Cornell Scale for Depression in Dementia, NPI-Q: Neuropsychiatric Inventory-Questionnaire, ZBI: Zarit Burden Interview, QOL: quality of life, PSAPs: personal sound amplification products, CP: communication partner, AR: aural rehabilitation, ALD: assistive listening device, DCHD: direct-to-consumer hearing devices 
tients from reporting real-world experience. For these reasons, the study results generally lack ecological validity and should not be generalized. Future studies should be conducted with robust study design, long-term outcome measurement, and more realistic OTC service-delivery model (e.g., self-selection of the device that is sold on the free market with no additional support) to more accurately represent the outcomes that DCHD can provide for people with hearing loss. Additionally, the incorporation of phone applications as amplification tools can be considered. Further research, and possibly the supplemental research of the two pilot studies discussed here, will provide further outcome verifications derived by more robust study designs.

\section{Conclusion}

The literature suggests positive outcomes of DCHD in older adults with hearing loss during short-term (i.e., less than one year) trials. Improvements in auditory ability, communicative function, social engagement, quality of life, and reduction of self-reported hearing disability were observed. Yet, a majority of studies conducted on this topic are of low quality of evidence. In addition, results may have been influenced by researcher and/or clinician involvement in choosing the devices and by provision of additional support (e.g., incorporation of a $\mathrm{CP}$, communication strategies training). The nature of direct-to-consumer method makes it challenging to design studies that will accurately represent outcomes for patients due to the extensive dissimilarities in patient journey and device selection options. Hence, additional research utilizing more robust study design, measuring long-term outcomes, alternative product options, and a more traditional direct-to-consumer delivery method should be conducted before generalization of benefits is applied to a wider population.

\section{Conflicts of interest}

The authors have no financial conflicts of interest.

\section{REFERENCES}

1) World Health Organization. Deafness and hearing, fact sheet [cited 2017 September 1]. Available from: http://www.who.int/mediacentre/factsheets/fs $300 / \mathrm{en} /$.

2) Blackwell DL, Lucas JW, Clarke TC. Summary health statistics for U.S. adults: national health interview survey, 2012. Vital Health Stat 2014;10:260.

3) National Institute on Deafness and other Communication Disorders (NIDCD). Quick statistics about hearing [cited 2017 September 1]. Available from: https://www.nidcd.nih.gov/health/statistics/quickstatistics-hearing \#6.

4) U.S. Census Bureau. The baby boom cohort in the United States: 2010 to 2060 [cited 2017 September 1]. Available from: https://www. census.gov/prod/2014pubs/p25-1141.pdf.

5) Abrams HB, Kihm J. An introduction to MarkeTrak IX: a new baseline for the hearing aid market. Hear Rev 2015;22:16.

6) Davis A, Smith P, Ferguson M, Stephens D, Gianopoulos I. Accept- ability, benefit and costs of early screening for hearing disability: a study of potential screening tests and models. Health Technol Assess 2007:11:1-294.

7) Kochkin S. MarkeTrak VIII: 25 year trends in the hearing health market. Hear Rev 2009;16:12-31.

8) Hougaard S, Ruf S. EuroTrak I: a consumer survey about hearing aids in Germany, France and the UK. Hear Rev 2011;18:12-28.

9) Knudsen LV, Oberg M, Nielsen C, Naylor G, Kramer SE. Factors influencing help seeking, hearing aid uptake, hearing aid use and satisfaction with hearing aids: a review of the literature. Trends Amplif 2010;14:127-54.

10) Arnold ML, Hyer K, Chisolm T. Medicaid hearing aid coverage for older adult beneficiaries: a state-by-state comparison. Health Aff (Millwood) 2017;36:1476-84.

11) Manchaiah V, Taylor B, Dockens AL, Tran NR, Lane K, Castle M, et al. Applications of direct-to-consumer hearing devices for adults with hearing loss: a review. Clin Interv Aging 2017;12:859-71.

12) Kochkin S. MarkeTrak VIII: utilization of PSAPs and direct-mail hearing aids by people with hearing impairment. Hear Rev 2010; 17:12, 14-16.

13) Warren E, Grassley C. Over-the-counter hearing aids: the path forward. JAMA Intern Med 2017;177:609-10.

14) Thomas KP. Are direct-to-consumer marketing and over-the-counter sale of hearing aids beneficial to patients with hearing loss? A provider's perspective. N C Med J 2017;78:109-10.

15) Cheng CM, McPherson B. Over-the-counter hearing aids: electroacoustic characteristics and possible target client groups. Audiology 2000;39:110-6.

16) Callaway SL, Punch JL. An electroacoustic analysis of over-thecounter hearing aids. Am J Audiol 2008;17:14-24.

17) Chan ZY, McPherson B. Over-the-counter hearing aids: a lost decade for change. Biomed Res Int 2015;2015:827463.

18) Smith C, Wilber LA, Cavitt K. PSAPs vs hearing aids: an electroacoustic analysis of performance and fitting capabilities. Hear Rev 2016;23:18.

19) Reed NS, Betz J, Lin FR, Mamo SK. Pilot electroacoustic analyses of a sample of direct-to-consumer amplification products. Otol Neurotol 2017;38:804-8.

20) Atkins D, Best D, Briss PA, Eccles M, Falck-Ytter Y, Flottorp S, et al. Grading quality of evidence and strength of recommendations. BMJ 2004;328:1490.

21) Brozek JL, Akl EA, Alonso-Coello P, Lang D, Jaeschke R, Williams $\mathrm{JW}$, et al. Grading quality of evidence and strength of recommendations in clinical practice guidelines. Part 1 of 3 . An overview of the GRADE approach and grading quality of evidence about interventions. Allergy 2009;64:669-77.

22) Mamo SK, Nirmalasari O, Nieman CL, McNabney MK, Simpson A, Oh ES, et al. Hearing care intervention for persons with dementia: a pilot study. Am J Geriatr Psychiatry 2017;25:91-101.

23) Nieman CL, Marrone N, Mamo SK, Betz J, Choi JS, Contrera KJ, et al. The Baltimore HEARS Pilot Study: an affordable, accessible, community-delivered hearing care intervention. Gerontologist 2017; 57:1173-86.

24) McPherson B, Wong ET. Effectiveness of an affordable hearing aid with elderly persons. Disabil Rehabil 2005;27:601-9.

25) Sacco G, Gonfrier S, Teboul B, Gahide I, Prate F, Demory-Zory M, et al. Clinical evaluation of an over-the-counter hearing aid (TEO First $(\mathbb{R})$ in elderly patients suffering of mild to moderate hearing loss. BMC Geriatr 2016;16:136.

26) Humes LE, Rogers SE, Quigley TM, Main AK, Kinney DL, Herring C. The effects of service-delivery model and purchase price on hearing-aid outcomes in older adults: a randomized double-blind placebo-controlled clinical trial. Am J Audiol 2017;26:53-79.

27) Reed NS, Betz J, Kendig N, Korczak M, Lin FR. Personal sound amplification products vs a conventional hearing aid for speech understanding in noise. JAMA 2017;318:89-90. 